09

\title{
Повышение чувствительности обнаружения метана в атмосферах экзопланет и туманностях спектроскопическими методами
}

\author{
(C) Ю.А. Толмачев, М.П. Иванов, В.А. Глухов \\ Санкт-Петербургский государственный университет, \\ 199034 Санкт-Петербург, Россия \\ e-mail: ytolmachev@gmail.com
}

(Поступило в Редакцию 3 июля 2017 г.)

Рассмотрены вопросы технического развития оптимальных методов обнаружения простых молекул в газовой фазе по колебательно-вращательным или вращательным спектрам путем одновременной регистрации совокупности линий молекулярной полосы с помощью гребенчатого частотного фильтра. Предложены два варианта реализации методов: на основе спектрометра с решеткой-эшелле при резко ограниченном числе элементов и с френелевской линзой (зеркалом) со специальным образом рассчитанными кольцевыми компонентами, выполняющей функции решетки. Образованный френелевский фильтр может быть хорошо согласован с параметрами оптики астрономических инструментов без потери их светосилы.

DOI: 10.21883/JTF.2018.03.45604.2414

\section{Введение}

Обнаружение простых молекул в атмосферах планет Солнечной системы и экзопланет, а также во внеземных газо-плазменных образованиях и туманностях имеет огромное значение не только с точки зрения поиска ответа на вопросы, связанные с происхождением жизни, но и для получения информации об атмосфере самой Земли [1]. Это существенно расширяет наше понимание химических и физических процессов в атмосфере Земли, что может способствовать предотвращению необратимых изменений условий существования человечества.

Спектроскопический метод является основным при дистанционной диагностике молекулярного состава атмосферных газов, и повышение чувствительности датчиков оптических газоанализаторов - это одна из постоянных задач современной техники и науки. Получены обзорные спектры атмосферы некоторых экзопланет в области до $10 \mu \mathrm{m}[2]$, при этом предполагается наличие в атмосфере молекул $\mathrm{H}_{2} \mathrm{O}, \mathrm{CO}, \mathrm{CO}_{2}$ [3], метана, цианидов [4] и других газов.

Основным инструментом служат спектрометры, предназначенные для регистрации широких участков спектра [5-10] с невысокой разрешающей силой $R=\lambda / \delta \lambda$. Например, в [5] авторы ограничиваются $R \approx 70$, а в $[6]-R \approx 130$. Параллельно на базе классических принципов конструирования спектрометров для решения астрофизических задач методами внеземной астрономии создаются универсальные системы с разрешением $R=(1.0-1.2) \cdot 10^{5}[7,8]$. Оценки показывают, что даже с применением наземных телескопов в ближайшем будущем можно будет обнаружить следы кислорода по молекулярным полосам в ближней инфракрасной области спектра [9]. Возможности экспериментального исследования расширятся при выносе приборов за пределы земной атмосферы.
Параллельно развиваются методы точного определения частот компонентов молекулярных спектров [10], основанные на их сравнении с „сеткой частот“ из набора эквидистантных линий лазерной генерации (astro-comb), полученной при комбинации гребенки лазерных линий с фильтром на основе интерферометра Фабри-Перо (см. [11] и ссылки в этой работе). Потенциальная точность подобного инструмента намного превосходит ширину линий молекулярного спектра, что вызывает сомнения в необходимости подобного усложнения техники (особенно принимая во внимание усилия, требуемые для улучшения самой структуры линий результирующей гребенки частот). Вместе с тем недостаточно внимания уделяется проблемам увеличения мощности сигнала, полученного при изучении вполне конкретного типа молекул, поиск которых осуществляется в опыте, что позволяет понизить порог обнаруживаемых концентраций. Как правило, правда, при этом приходится отказываться от универсальности самой системы измерений.

Настоящая работа посвящена развитию оптимизационного подхода к решению общей задачи обнаружения $[12,13]$ и измерению малых или даже исчезающе малых концентраций молекул с максимальным использованием априорной информации об их спектре. Этот подход основан на общих следствиях теории информации и обнаружения сигнала. Вся совокупность компонентов спектра вещества (или максимально возможной его части) рассматривается как единый „сигнал“, несущий информацию о концентрации данного вещества. Сущность и новизна концепции заключена в словах „вся совокупность“. В применении к задаче обнаружения простых молекул, о которых шла речь выше, по их вращательным или колебательно-вращательным спектрам мы предлагаем использовать в одном акте измерения не одну линию молекулы, а максимально полный набор спектральных составляющих исследуемой молекулы. Метод решения 
поставленной задачи базируется на том, что спектры таких молекул состоят из почти эквидистантных линий.

Работа построена по следующей схеме: сначала приводятся основные математические предпосылки, составляющие фундамент работы; затем рассматривается конкретный пример структуры системы, позволяющей реализовать предлагаемую схему; приводятся данные математического моделирования подавления систематических спектральных помех и результаты эксперимента, согласующегося с принятой концепцией и подтверждающего ее корректность; наконец, дается одна из схем возможной реализации спектральной оптической системы, согласующейся с особенностями работы астрофизических инструментов.

\section{Обоснование методики}

Среди задач молекулярного спектрального анализа выделим одну, как наиболее актуальную: обнаружение молекул некоторого наперед заданного вида. Объектом нашего внимания являются самые простые молекулы с хорошо разрешенной структурой вращательного или ровибронного спектра. В качестве конкретного примера рассматривается молекулярная полоса метана, лежащая в инфракрасной (ИК) области прозрачности земной атмосферы вблизи $3000 \mathrm{~cm}^{-1}$ (рис. 1). Ветви $\mathrm{P}$ и R этой полосы при нормальных условиях включают в себя большое число линий, имеющих близкую по интенсивности величину, причем вид распределения амплитуд по частотам может быть заранее рассчитан [14]. Уровень шумов фотоприемника в ИК и микроволновом диапазоне практически не зависит от величины измеряемого светового потока, а время эксперимента обычно ограничено, особенно в условиях астрофизического эксперимента. В сочетании перечисленные особенности позволяют считать задачу обнаружения метана спектральными методами эквивалентной хорошо изученной задаче обнаружения известного сигнала в шуме. Повторим, что под „сигналом“ мы будем понимать всю совокупность линий полосы. Такой подход в решении некоторых спектроскопических задач, как показывает опыт [15], позволяет существенно повысить надежность результатов измерений.

Если рассматривать выделение и регистрацию интенсивности $k$-той компоненты полосы как действие одного канала передачи информации о концентрации молекулы, то для оценки количества информации $S_{k}$, переданной в этом канале за время $T_{k}$, воспользуемся формулой Шеннона для канала связи с шумами [16]:

$$
S_{k} \propto T_{k} \log \left(1+P_{s k} / P_{n k}\right) \text {, }
$$

где $P_{s k}-$ мощность сигнала, соответствующего этой компоненте, а $P_{n k}-$ мощность шума. Предположим, что уровень шума в каждом $k$-том канале одинаков $\left(P_{s 1}=P_{s 2}=\cdots=P_{s M} \equiv P_{s}\right)$, тогда, как показано в $[12$,
$13]$, при малом уровне сигнала $\left(P_{s k} / P_{n k} \ll 1\right)$ и фиксированном полном времени эксперимента измерения $T_{0}$ из соотношения (1) следует:

$$
S \propto \frac{T_{0}}{M} \sum_{k=1}^{M}\left(P_{s} / P_{n}\right)=T_{0}\left(P_{s} / P_{n}\right) .
$$

Это соотношение показывает, что количество информации, полученной в результате подобного опыта, не зависит от числа использованных линий, достаточно все время наблюдения затратить на измерение только одной линии. Именно такой вариант реализуется в большинстве имеющихся сегодня систем спектрального мониторинга.

Иначе выглядят результаты оценки при многоканальном методе регистрации, когда единовременно измеряется суммарная мощность излучения всех линий полосы, имеем

$$
S \propto \sum_{k=1}^{M}\left(E_{s k} / P_{n k}\right)=T_{0} \sum_{k=1}^{M}\left(P_{s k} / P_{n k}\right) \approx M T_{0}\left(P_{s} / P_{n}\right) .
$$

Как мы видим, количество информации в этом случае прямо пропорционально числу использованных линий и может возрасти более чем на порядок величины, аналогично „выигрышу Фелжета“ в фурье-спектроскопии [17]. Такой вариант системы обнаружения эквивалентен применению „гребенчатого фильтра“ в радиотехнике для оптимизации обнаружения сигнала, известного по форме.

Таким образом, создание спектрального фильтра, обеспечивающего максимальную вероятность обнаружения заданного по каким-то соображениям конкретного вещества, должно отвечать двум условиям:

- во-первых, фильтр должен пропустить компоненты спектра только этого вещества, охватывая максимально возможное их число;

- во-вторых, фильтр должен обеспечить суммирование парциальных сигналов отдельных компонентов без увеличения результирующего шума.

\section{Задача обнаружения метана}

Как показывает рис. 1, расстояние между линиями выбранной полосы метана почти постоянно и равно $10 \mathrm{~cm}^{-1}$, причем в нормальных условиях не менее десятка из них имеют сравнимую по величине интенсивность (показатель поглощения) - обстоятельство, имеющее важное значение для развиваемого метода. Уменьшение температуры приводит к сужению функции распределения интенсивностей компонентов полосы, но одновременно возрастает интенсивность линий с малым вращательным квантовым числом, что оказывается важным при исследовании содержания газа в туманностях.

Задачу, следовательно, можно свести к созданию фильтра эквидистантных частот, совпадающих с частотами компонентов молекулярной полосы. Подобный 


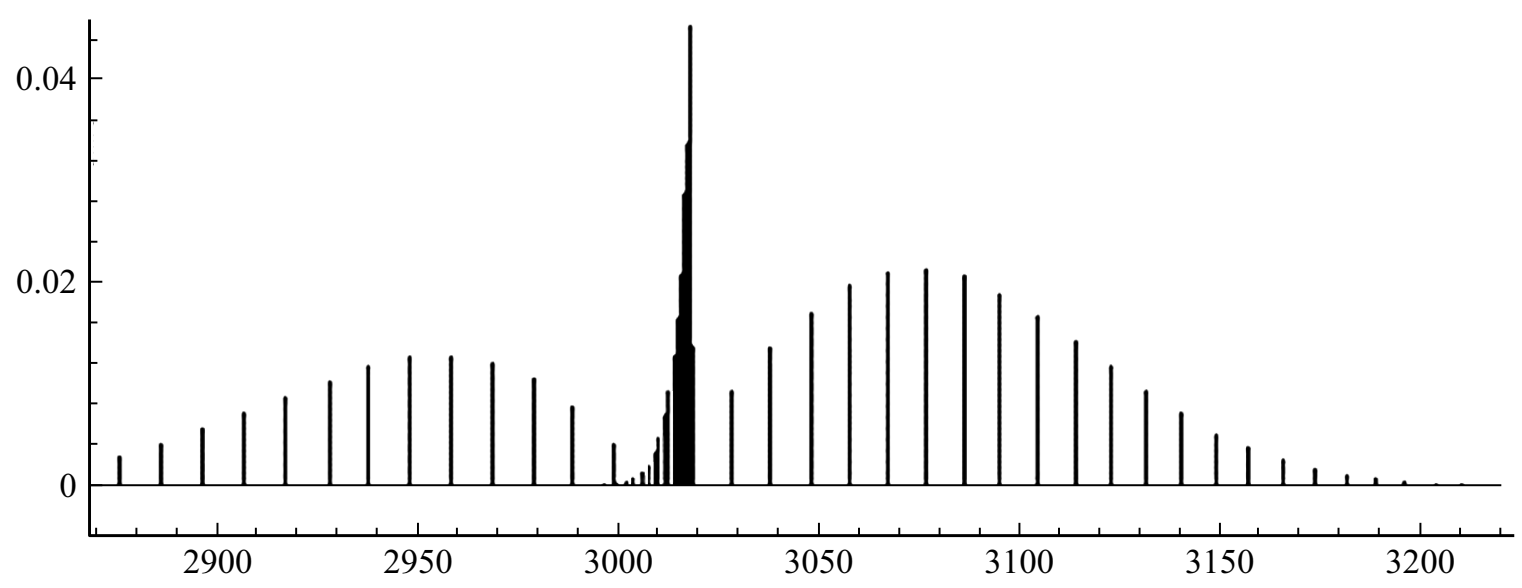

Рис. 1. Колебательно-вращательный спектр метана. Расчет по [14].

фильтр был рассмотрен в работе [18], авторы использовали давно известный эффект преобразования излучения с непрерывным спектром в так называемый „канавчатый“ вследствие двулучевой интерференции [19]. Спектр пропускания интерферометра характеризуется набором эквидистантных (в шкале частот) максимумов и минимумов. В качестве примера в этой работе приведен спектр теплового источника, прошедшего через двулучевой интерферометр Майкельсона. Регулируя разность хода в плечах интерферометра, можно добиться совпадения положения максимумов с линиями поглощения (излучения) молекул. Обладая универсальностью, фильтр, использующий двулучевой интерферометр, не приведет к существенному увеличению сигнал/шум при измерениях, так как ширина линий его пропускания составляет $1 / 2$ расстояния между максимумами. Значительно более перспективным является применение для этих целей эффектов интерференции многих пучков.

Принцип действия всех интерференционных устройств основан на суммировании копий исходной световой волны, сдвинутых во времени на фиксированную величину. Максимальное пропускание для излучения с длиной волны $\lambda_{k}$ наблюдается при выполнении условия

$$
b \Gamma=m \lambda_{k}, \text { при } m= \pm 1, \pm 2, \pm 3 \ldots
$$

В этом соотношении $b \Gamma-$ константа, заданная конструкцией и геометрией системы, причем обычно $b$ определяется параметрами самого интерференционного устройства, а фактор $\Gamma-$ условиями опыта, в частности, углом падения исходного светового пучка и углом наблюдения прошедшего излучения. В случае плоской отражательной дифракционной решетки, например, $b-$ ширина ее штрихов, $\Gamma=\sin \varphi+\sin i$, где $\varphi$ и $i-$ углы падения и дифракции пучка, для интерферометра Фабри-Перо $b$ есть удвоенная толщина интерферометpa, а $\Gamma=\cos \varphi$, и т.д. Целое число $m$ есть „порядок“ интерференции, оно показывает на сколько полных периодов (на сколько длин волн $\lambda_{k}$ ) сдвигается в интер- ферометре одна копия исследуемой волны относительно другой.

Если переписать соотношение (3) в частотах, выраженных в обратных сантиметрах $\left(\sigma_{K}=1 / \lambda_{k}\right)$, получим $b Г \sigma_{k}=m$ или

$$
\sigma_{k}=m(1 / b \Gamma)
$$

Следовательно, в шкале частот соседние максимумы пропускания интерференционной системы, которым соответствует $\Delta m \pm 1$, расположены эквидистантно, а расстояние между ними $\Delta \sigma$ определяется геометрическими параметрами используемой системы и равно

$$
\Delta \sigma=1 / b \Gamma
$$

По форме (5) совпадает с соотношением для „интервала частот, свободного от переложений“ в спектральных приборах. Его физический смысл состоит в том, что монохроматическое излучение разных порядков дифракции с частотами, отстоящими друг от друга на $\Delta \sigma$, проходят через интерференционную систему в одном и том же направлении, световые потоки всех компонентов полосы, распространяющиеся в этом направлении, суммируются. В традиционных спектральных приборах этот факт рассматривается как помеха частотному анализу, но можно использовать его для увеличения амплитуды измеряемого сигнала.

Расстояние между компонентами вращательного или колебательно-вращательного спектра молекулы не остается постоянным при изменении вращательного квантового числа. Следовательно, при оптимизации процесса обнаружения с помощью многолучевой интерференционной системы необходимо решить противоречивую задачу: ширина спектрального контура пропускания системы для монохроматического колебания (ее аппаратной функции) должна быть максимально мала, чтобы минимизировать возможное влияние мешающего излучения, но одновременно она должна оставаться настолько большой, чтобы охватить значительное число линий полосы. Решение сформулированной задачи лежит на 
пути искусственного увеличения ширины аппаратной функции. Один из возможных путей - уменьшение числа интерферирующих пучков.

Анализ совокупности частот $\mathrm{P}$ и R-ветвей, показанных на рис. 1 полос метана, дает следующие усредненные значения для центральной частоты $\sigma_{0}$, расстояния между компонентами $\Delta \sigma$ и вариации этого расстояния $\delta(\Delta \sigma)$ :

$$
\sigma_{0}=3030 \mathrm{~cm}^{1}, \Delta \sigma=9.7 \mathrm{~cm}^{-1}, \delta(\Delta \sigma)=0.17 \mathrm{~cm}^{-1} .
$$

Последнее значение по крайней мере на порядок превышает ожидаемый допплеровский сдвиг линий полосы, связанный с движением различных областей нашей Галактики относительно Земли, т.е. при исследовании спектров объектов в пределах Галактики отпадает необходимость в перестройке частот фильтра.

Рассмотрим пути возможной реализации соответствующего полихроматического фильтра.

\section{Гребенчатый фильтр на основе плоской дифракционной решетки}

Возьмем за основу фильтр оптических частот, построенный по схеме автоколлимационного спектрального прибора с дифракционной решеткой. Значения углов $i$ и $\varphi$ совпадают, формула (4) приобретает вид

$$
\sigma_{k}=k /(2 b \sin \varphi) \text {. }
$$

Зададим угол падения света на решетку равным $60^{\circ}$, номер порядка дифракции для центра полосы найдем из условия

$$
k_{0}=\frac{\sigma_{0}}{\Delta \sigma}=\frac{3030}{9.7} \approx 312 .
$$

Для дальнейших оценок будем полагать $k_{0}=310$, тогда при $\varphi=60^{\circ}$, ширина штриха решетки оказывается равной $b=0.59 \mathrm{~mm}$. Если принять величину $\delta(\Delta \sigma)=0.17 \mathrm{~cm}^{-1}$ в качестве предельной разрешающей способности решетки, то необходимое число ее штрихов $N$ можно найти из известного соотношения $R=\sigma_{0} / \delta(\Delta \sigma)=k N$, откуда следует $N=57$, и ширина решетки оказывается всего $33 \mathrm{~mm}$.

Приведенные оценки показывают, что дифракционная решетка с подобными параметрами есть не что иное как решетка эшелле, работающая в очень высоких порядках, и ее изготовление видится достаточно простым. Высокие значения порядков дифракции видимого излучения в современной технике спектрального приборостроения не представляют собой нечто новое даже для видимого диапазона длин волн (см., например, [20], где $k=140$ ). Профилированные штрихи эшелле позволяют концентрировать в заданном направлении достаточно узкий спектральный интервал, что обеспечивает предварительную квазимонохроматизацию излучения. Этот интервал дополнительно может быть ограничен использованием соответствующих интерференционных фильтров [21].

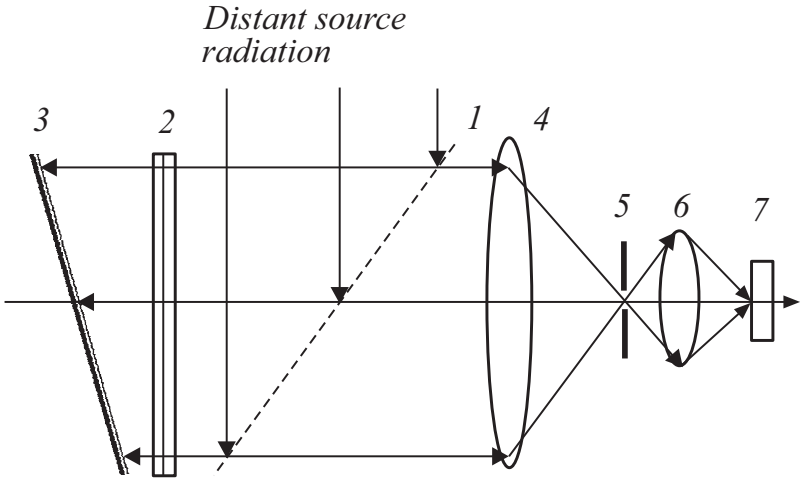

Рис. 2. Схема фильтра на основе дифракционной решетки. 1 - полупрозрачное зеркало, 2 - интерференционный фильтр и аподизирующая диафрагма, 3 - дифракционная решеткаэшелле, 4 - конденсор, 5 - щель спектрометра, 6 - согласующий объектив, 7 - фотоприемник и система обработки сигнала.$$
\text { ||l|||||||||||||| }
$$$$
2.7
$$$$
2.8
$$$$
\sigma, 10^{3} \mathrm{~cm}^{-1}
$$

Рис. 3. Отклик спектрометра с решеткой-эшелле на излучение со сплошным спектром (нормировано).

Возможная оптическая схема такого гребенчатого фильтра представлена на рис. 2, он построен по типовой схеме автоколлимационного спектрометра. На фотоприемник проходят все компоненты спектра, удовлетворяющие условию (4), т.е. осуществляется суммирование сигналов всех частотных компонентов полосы. Безусловно, возможно использование любых иных вариантов оптических схем, применяемых в классических приборах для спектрального анализа.

Отметим, что применение известных методов аподизации контура, например, путем размещения ромбической диафрагмы на апертуре решетки, позволяет существенно увеличить селективность фильтра. Потеря в геометрическом факторе и увеличение ширины его аппаратного контура компенсируются при этом соответствующим увеличением размеров решетки с сопутствующим увеличением числа штрихов. Увеличение контраста сформированного спектра в результате аподизации приведет также к повышению эффективности подавления спектра помехи.

Нами был проведен эксперимент, в котором использовалась установка эшелле $60 \mathrm{~mm}^{-1}$ в режиме, близком к автоколлимационному с медленным сканированием спектра путем поворота решетки. Регистрация области 
спектра, близкой к $2.8 \mu \mathrm{m}$, осуществлялась с помощью охлаждаемого $\mathrm{PbS}$ фотосопротивления, источником света служил глобар. Сигнал регистрировался непосредственно с фотосопротивления с помощью осциллографа C1-30, обладающего длительным послесвечением экрана. Результат регистрации, приведенный на рис. 3 , после оцифровки показывает, что основные предположения о наличии у подобного спектрального фильтра гребенки равноотстоящих максимумов пропускания выполняются.

Приведенные выше оценки были сделаны для входной апертуры в виде предельно тонкой входной щели прибора. При использовании щели конечной ширины некоторую проблему может составить сам эффект переложения порядков дифракции, использованный при построении фильтра, так как в фокальной плоскости формируется огромное число изображений щели в близких по величине длинах волн молекулярной полосы. В рассматриваемом случае угловой интервал между соседними порядками дифракции составляет всего $3 \cdot 10^{-4}$, что соответствует $150 \mu \mathrm{m}$ при фокусном расстоянии коллиматора $f=500 \mathrm{~mm}$. Следовательно, ширина выходной щели должна быть не более $75 \mu \mathrm{m}$ для такого варианта. Полученное значение в несколько раз превосходит нормальную ширину щели для рассматриваемых параметров прибора, но в то же время ограничивает величину светового потока, проходящего через фильтр.

\section{Влияние систематических помех, вызванных молекулами с близкими частотами}

Для проверки одного из свойств подобного гребенчатого фильтра, а именно наличия эффекта подавления спектров иных молекул с близкими по величине частотами полос, мы произвели моделирование ситуации, когда при равенстве интенсивностей исследуемой и мешающей полос их центры совпадают, но вращательные постоянные различаются. Очевидно, что в этом случае возможно случайное совпадение частот пропускания фильтра и компонентов мешающей полосы. Уменьшить влияние помехи можно, прибегнув к методам модуляционной спектроскопии [22], вводя небольшую периодическую перестройку по частоте, допустим, периодическим малым поворотом эшелле в направлении дисперсии.

В качестве примера на рис. 4 показаны модуляционные характеристики - зависимость амплитуды выходного сигнала от смещения частоты настройки фильтра от резонанса - для основной молекулы и мешающей полосы, равной ей по интенсивности, причем предполагается, что распределение интенсивностей молекулыпомехи однородно по частоте. Вращательная постоянная помехи принята равной 0.8 от постоянной исследуемой молекулы. При таких параметрах, как видно из этого рисунка, фоновая составляющая помехи уменьшается приблизительно в 6 раз, причем глубина ее модуляции на порядок величины меньше, чем основной. Величина

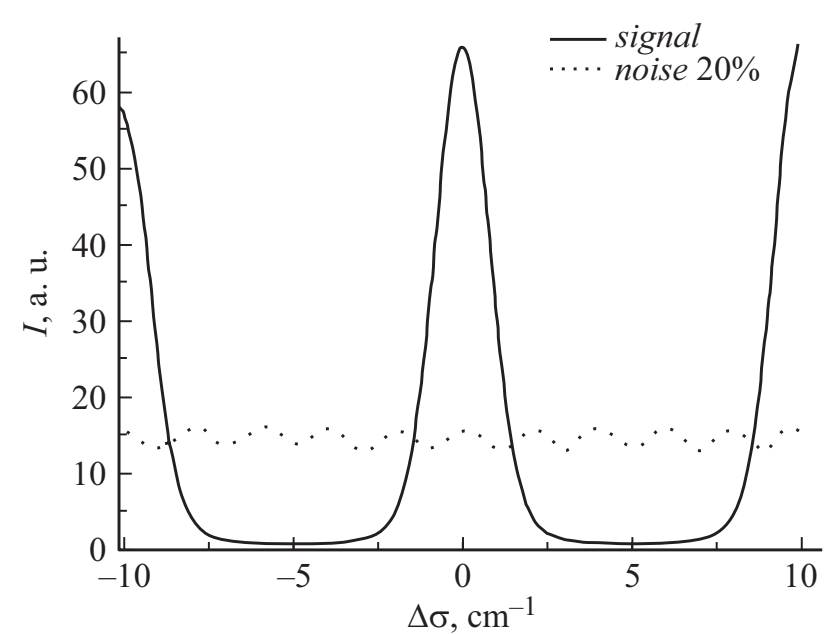

Pис. 4. Зависимость амплитуды выходного сигнала фильтра от величины смещения частоты отстройки от точного резонанса (модуляционная кривая) для фильтра с равномерным распределением пропускания. Сплошная кривая - сигнал основной молекулы, пунктир - сигнал помехи равной интенсивности при равномерном распределении интенсивностей в полосе и отличии вращательной постоянной на $20 \%$.

фоновой постоянной сигнала помехи и глубина осцилляций уменьшаются приблизительно обратно пропорционально отношению вращательных постоянных. Отметим также два дополнительных обстоятельства. Во-первых, обнаружена большая роль побочных максимумов аппаратной функции, уменьшить их можно введением упомянутой выше аподизации аппаратного контура системы. Во-вторых, рис. 4 показывает, что сигнал помехи содержит высокочастотную компоненту, амплитуду которой при периодической модуляции величины отстройки от резонанса можно дополнительно уменьшить с помощью низкочастотного фильтра, подавляющего соответствующие компоненты тока фотоприемника.

Таким образом, данный раздел работы убедительно демонстрирует фундаментальные достоинства устройств, основанных на многолучевой интерференции, в решении задачи одновременной фильтрации многих компонентов молекулярной полосы.

\section{Фильтр на основе зеркала (линзы) Френеля}

Системы обнаружения молекул в астрофизических задачах работают с источниками излучения, имеющими малые угловые размеры. Следовательно, их линейные размеры на входе описанного выше гребенчатого спектрального фильтра также будут малы соответственно, невелики могут быть и размеры входной апертуры (в частности, высота щели при использовании дифракционной решетки). Целесообразно, следовательно, рассмотреть иные возможности реализации оптического гребенчатого фильтра в свете сформулированных выше 
к нему требований. Обратим внимание на то, что фурьеобразу сигнала на выходе гребенчатого фильтра частот соответствует периодическое повторение этого сигнала.

Сейчас для преобразования одиночного ультракороткого импульса в серию эквидистантных во времени в пространстве копий используются конструкции типа эшелона Майкельсона $[23,24]$. Одной из систем, обеспечивающих аналогичное повторение и используемых для решения задач поиска и изучения свойств экзопланет, сегодня является стремительно развивающаяся техника, имеющая в своей основе интерферометр Фабри-Перо (ИФП). Используемый как спектральный прибор высокой разрешающей силы ИФП позволяет с высокой точностью измерять допплеровские сдвиги частот слабых линий [25-27]. Такие устройства пока применяются только в видимой и ближней ИК области спектра. Являясь деликатным устройством, требующим регулярной настройки и ориентированным на использование в системах с высокой разрешающей силой, ИФП тем не менее может с успехом применяться для решения задач обнаружения молекул описанным выше методом. Необходимость расширения аппаратного контура, правда, приводит к одновременному понижению контраста интерференционной картины вследствие увеличения пропускания в области минимумов аппаратной функции. Соответственно снижаются возможности применения модуляционной спектроскопии.

Несмотря на повышенные требования к механической стабильности ИФП, несомненным их достоинством является цилиндрическая симметрия, позволяющая оптимизировать сочетание фильтра с круглым зрачком абсолютного большинства современных астрофизических наблюдательных систем. В качестве альтернативы ИФП рассмотрим иную схему, позволяющую сохранить цилиндрическую симметрию интерференционного поля и лишенную указанных выше недостатков, а именно линзу Френеля. Экспериментальное исследование преобразования фемтосекундного импульса линзой Френеля [28] показало, что при выполнении определенных требований к конструкции линзы сигнал в фокальной точке состоит из серии равноотстоящих во времени копий входного импульса. Соответственно спектр сигнала распадается на равноотстоящие в пространстве частот линии.

Для ИК или микроволновой области спектра целесообразнее использовать зеркальную систему, состоящую из системы примыкающих друг к другу кольцевых вогнутых софокусных концентрических зеркал. Для концентрации излучения в технике используются подобные системы зеркал одинаковой ширины (рис. 5), которые называются „френелевский отражатель“ (Fresnel reflector) (в отличие от „зеркал Френеля“ - известной системы двух зеркал, применяющейся для создания и изучения системы интерференционных полос). Ориентированный только на увеличение энергетических характеристик такой „френелевский отражатель“ не обеспечивает реализацию интерференционных свойств, необходимую для

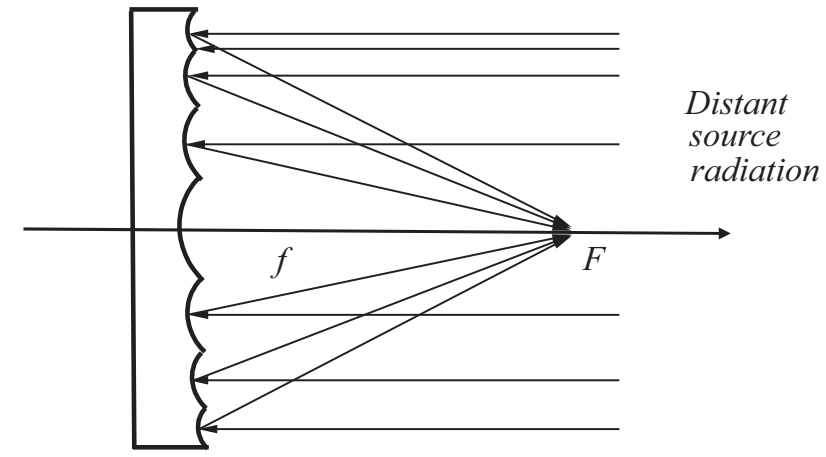

Рис. 5. Схема френелевского отражателя с фокусным расстоянием $f$. Показано сечение зеркала плоскостью симметрии.

создания гребенчатого фильтра, согласованного по частотам со спектром молекулы.

Оптический гребенчатый фильтр должен, во-первых, обеспечить возможность интерференции всех вторичных волн, сформированных из падающей на него волны, для этого они должны сходиться в одной точке - фокусе всех компонентов зеркала (или линзы). Во-вторых, разность хода $\Delta l$ между отдельными сходящимися пучками должна быть постоянна, откуда для расстояния $l_{k}$ между точкой фокуса и границей $k$-того компонента получаем простое соотношение

$$
l_{k}=f+k \Delta l .
$$

При этом для образования пучности в фокусе необходимо выполнить требование $\Delta l=m \lambda_{0}$ или эквивалентного ему

$$
\sigma_{0} \cdot \Delta l=m .
$$

Число $m$ выбирается, как и раньше, в соответствии с необходимой разностью частот между линиями полосы и совпадает с величиной $k_{0}$, введенной нами ранее:

$$
m=k_{0}=\frac{\sigma_{0}}{\Delta \sigma}=\frac{3030}{9.7} \approx 312 .
$$

При освещении кольцевого сферического (в идеальном случае - параболического) сегмента вогнутого зеркала плоской волной отраженная волна имеет форму сферического кольцевого сегмента, сходящегося в точку фокуса. Чтобы амплитуды отраженных волн в этой точке были одинаковы, необходимо дополнительно выполнить условие равенства площадей колец. Для примыкающих друг к другу колец, в частности, оно имеет вид

$$
r_{k+1}^{2}-r_{k}^{2}=\text { const. }
$$

Используя импульсный метод анализа, в работах $[28,29]$ мы рассмотрели особенности подобной конструкции в применении к линзе Френеля и отметили, что в этом случае происходит когерентное сложение волн. В итоге, несмотря на то что дифракционная картина в фокальной плоскости от каждого из колец описывается функцией, пропорциональной функции Бесселя 
Внешние радиусы кольцевых зеркал для $f=500 \mathrm{~mm}$

\begin{tabular}{c|c}
\hline$k$ & $r_{k}, \mathrm{~mm}$ \\
\hline 0 & 0 \\
1 & 30.7 \\
2 & 43.4 \\
$\ldots$ & $\ldots$ \\
19 & 135 \\
20 & 138 \\
$\ldots$ & $\ldots$ \\
56 & 235 \\
57 & 23.8
\end{tabular}

$J_{0}$, а не $J_{1}$, суммарные дифракционные свойства такой линзы для излучения с длиной волны $\lambda_{0}$ совпадают со свойствами обычной совершенной линзы:

- угловые размеры дифракционного пятна есть $\delta \varphi=1.22 \lambda_{0} / D$, где $D-$ внешний диаметр всей линзы;

- амплитуда волны в центре растет пропорционально полной площади линзы, т.е. пропорционально $D^{2}$ (соответственно интенсивность в центре увеличивается пропорционально $D^{4}$ ).

Это утверждение в полной мере может быть распространено и на „когерентный“ френелевский отражатель, удовлетворяющий условиям (6) и (7). Количество компонентов подобного отражателя $-N-$ рассчитывается так же, как и необходимое число штрихов решетки эшелле:

$$
N=\frac{\sigma_{0}}{k_{0} \delta(\Delta \sigma)}
$$

В качестве примера приведем (см. таблицу) результаты расчета некоторых радиусов кольцевых линз (в том числе - последнего из полученного выше значения $N=57$ ) для рассмотренного выше варианта обнаружения молекул метана в атмосфере при нормальных условиях. Достаточно при этом задать фокусное расстояние и определить тип конструкции зеркала. В частности, предполагается, что центральная часть зеркала не содержит отверстия, а фокусные расстояния колец равны $500 \mathrm{~mm}$.

Для применения техники модуляционной спектроскопии [22] перестройка по частотам может осуществляться периодическим смещением диафрагмы, вырезающей центральный дифракционный максимум, из точки фокуса вдоль оптической оси (или иным описанным в литературе методом). При этом весь набор расстояний $l_{k}$ изменяется, и свойства резонансности настройки фильтра и спектра поглощения нарушаются. Такое смещение может осуществляться как механическим перемещением приемника, так и перемещением его изображения, например, с помощью установленной в пучке поворачивающейся прозрачной плоскопараллельной пластинки.

Очевидно, что в реальном зеркальном фильтре центральная часть светового пучка будет перекрыта фотоприемником. Исключение центральной части приведет к некоторому увеличению всех радиусов элементарных зеркал, но конечные внешние размеры зеркала увеличатся незначительно. Гребенчатый фильтр, построенный на основе когерентного френелевского отражателя, должен хорошо согласовываться с большинством существующих телескопических систем и обеспечивать максимальное использование светового потока. Заметим, что применение вариации радиусов кольцевых зеркал с целью аподизации контуров пропускания фильтра приведет к изменению условий сложения амплитуд волн в окрестности фокальной точки. Следствием будет отступление от тождественности такой системы обычному вогнутому зеркалу и существенное изменение формы контуров аппаратной функции, поэтому наиболее предпочтительным методом является изменение эффективного коэффициента отражения элементарных кольцевых зеркал.

\section{Заключение}

Каждая из рассмотренных двух систем - с решеткойэшелле и с когерентным френелевским отражателем по основным своим свойствам не предназначена для универсального решения задачи обнаружения любых молекул. Они нацелены на спектральное обнаружение в данном опыте молекул только заданного конкретного вида. Обе системы обладают весьма своеобразным набором достоинств и недостатков. Так, например, применение решетки-эшелле при исследованиях спектров внеземных источников является привычным инструментом в практике астрофизики и не требует какого-то принципиального пересмотра технической реализации спектрометра. Весьма вероятно также, что даже переход от одного типа молекул к другому не потребует замены решетки. Вместе с тем традиционная геометрия решетки не оптимальна с точки зрения использования ее площади для увеличения светового потока. В этом отношении применение когерентного френелевского отражателя (или когерентной линзы Френеля) имеет преимущество. Полученные оценки габаритов соответствующей системы не представляют чего-то особенного для телескопических систем, используемых в практике астрофизических измерений.

Рассмотренные нами две схемы оптических систем, предназначенных для обнаружения простых молекул по их колебательно-вращательным спектрам, не исчерпывают возможности развития основанных на многолучевой интерференции устройств. В этой связи достаточно вновь упомянуть ИФП, также обладающий набором эквидистантных по частотам линий пропускания. Вместе с тем предложенные устройства обладают потенциальным преимуществом, связанным не только с простыми методами управления формой контура линий пропускания, но также с возможностью дополнительной оптимизации общей структуры спектра. Например, введение в оптический канал дополнительного двулучевого интерферометра позволяет приближенно согласовать форму распределения амплитуд пропускания с распределением 
интенсивности линий молекулярной полосы - создать „оптимальный“ (в терминах решения задач обнаружения сигнала) фильтр. В частном случае решетки-эшелле такого же эффекта можно достичь путем оптимизации формы распределения индикатрисы рассеяния излучения одним штрихом решетки.

Работа поддержана РНФ (проект 17-19-01097).

\section{Список литературы}

[1] Кораблев О.И. // УФН. 2005. Т. 175. № 6. С. 655-664.

[2] Knutson H.A., Benneke B., Deming D., Homeier D. // Nature. 2014. Vol. 505. P. 66-68. doi:10.1038/nature12887.

[3] Swain M.R., Vasisht G., Tinetti G. et al. // Astrophys. J. Lett. Vol. 690. N 2. L114-L117.

[4] Tsiaras A., Rocchetto M., Waldmann I.P. et al. // arXiv:1511.08901v2 [astro-ph.EP]. doi: $10.3847 / 0004-637 \mathrm{X} / 820 / 2 / 99$.

[5] Deming D., Wilkins A., McCullough P. et al // Astrophys. J. 2013. Vol. 774. N 2. P. 95. doi:10.1088/0004-637X/774/2/95.

[6] Mandell A.M., Haynes K., Sinukoff E. et al. // Astrophys. J. 2013. Vol. 779. № 2. P. 128.

[7] Richter M.J., Lacy J.H., Jaffe D.T. et al. $\mathrm{http}: / /$ www.as.utexas.edu/astronomy/research/exes/kona_exes.pdf

[8] Tokunaga A.T., Bond T., Elias J. et al. http://www.ifa.hawaii.edu/ tokunaga/Tokunaga06spie6269.pdf.

[9] Kawahara H., Matsuo T., Takami T. et al. // Astrophys. J. 2012. Vol. 758. N 1. P. 13. doi: 10.1088/0004-637X/758/1/13.

[10] Phillips D.F., Glenday A.G., Li Chih-Hao et al. // Optics Express. 2012. Vol. 20. N 13. P. 13711-13726.

[11] Probst R.A., Steinmetz T., Wilken T. et al. // Opt. Express. 2013. Vol. 21. N 10. P. 11670-11687. doi: 10.1364/OE.21.011670.

[12] Пермякова Е.С., Толмачев Ю.А. // Химическая физика. 2015. Т. 34. № 8. С. 78-82. (Permyakova E.S., Tolmachev Yu.A. Russ. J. Phys. Chem. B. 2015. Vol. 9. N 4. P. 578-581.) doi: 10.1134/S1990793115040284.

[13] Permyakova E.S., Tolmachev Yu.A. // Opt. Memory and Neural Networks (Information Optics). 2015. Vol. 24. N 1. P. 48-53. doi:10.3103/S1060992X15010075.

[14] PGOPHER version 8.0, C M Western, 2014, University of Bristol Research Data Repository. doi: 10.5523/bris.huflggvpcuc1zvliqed497r2.

[15] Ren M., Dong M., Liu J. // Energies. 2016. Vol. 9. N 3. P. 152. doi: 10.3390/en9030152.

[16] Shannon C.E // Bell System Tech. J. 1949. Vol. 27. P. 379423, 623-656.

[17] Fellgett P.B. // J. Opt. Soc. Am. 1949. Vol. 39. P. 970-976. doi: $10.1364 /$ josa.39.000970.

[18] Лукин К.А., Татьянко Д.Н., Мачехин Ю.П. // Міжнародний науковотехнічний журн. „Світлотехніка та Електроенергетика“" 2011. № 3. С. 26-30.

[19] Борн М., Воль $\varnothing$ Э. Основы оптики. Пер. с англ. под ред. Г.П. Мотулевич. М.: Наука, 1970. 856 с.

[20] Электронный ресурс. Режим доступа: http://www.rusnanonet.ru/download/equipment/ICPE9000.pdf.

[21] Электронный ресурс. Режим водоступа: http://www.ibs-st-petersburg.com/led_2html

[22] Кардона М. Модуляционная спектроскопия. Пер. с англ. / Под ред. А.А. Каплянского, М.: Мир, 1972.
[23] Katz J., Boni R., Rivlis R. et al. // Rev. Scientif. Instrum. 2016. Vol. 87. N 11. P. E535-1-3. doi: 10.1063/1.4961090.

[24] Noe II G.T., Katayama I., Katsutani F. et al. // Opt. Expres. 2016. Vol. 24. N 26. P. 30328-30337. http://dx.doi.org/10.1364/OE.24.030328.

[25] Glenday A.G., Li Chin-Hao, Langellier N. et al. // Optica. 2015. Vol. 2. N 3. P. 250-254. http://dx.doi.org/10.1364/OPTICA.2.000250.

[26] Li Chih-Hao, Glenday A.G., Benedick A.J. et al. // Opt. Expres. 2010. Vol. 18. N 12. P. 13239-13249.

[27] Chang G., Li Chih-Hao, Phillips D.F. et al. // Opt. Expres. 2012. Vol. 20. N 22. P. 24987-25013.

[28] Permyakova E.S., Statsenko T.V., Tolmachev Yu.A. // Opt. Memory and Neural Networks (Information Optics). 2012. Vol. 21. N 2. P. 63-69.

[29] Бартч Ш., Стаценко Т., Толмачев Ю. // Proceedings of the 8-th International Conference „HOLOEXPO-2011“, 29 September - 1 October, Minsk, Belarus. C. 467-470. (Bartsch S., Statsenko T., Tolmachev Yu. // Proceedings of the 8-th International Conference „HOLOEXPO-2011“, 29 September - 1 October, Minsk, Belarus. P. 470-471). 